\title{
Conductivity of dielectric and thermal atom-wall interaction
}

\author{
G. L. Klimchitskaya* and V. M. Mostepanenko ${ }^{\dagger}$ \\ Center of Theoretical Studies and Institute for Theoretical Physics, Leipzig University, D-04009, Leipzig, Germany
}

\begin{abstract}
We compare the experimental data of the first measurement of a temperature dependence of the Casimir-Polder force by Obrecht et al. [Phys. Rev. Lett. 98, 063201 (2007)] with the theory taking into account small, but physically real, static conductivity of the dielectric substrate. The theory is found to be inconsistent with the data. The conclusion is drawn that the conductivity of dielectric materials should not be included in the model of the dielectric response in the Lifshitz theory. This conclusion obtained from the long separation measurement is consistent with related but different results obtained for semiconductors and metals at short separations.
\end{abstract}

PACS numbers: $42.50 . \mathrm{Nn}, 77.22 . \mathrm{Ch}$

The Casimir-Polder force [1] acts between rarefied atoms and a wall. It originates from zero-point and thermal fluctuations of the electromagnetic field. At separations $x$ less than a few nanometers (but larger than a few angstroms) the interaction between an atom and a wall is of nonrelativistic character and takes the form of the nonretarded van der Waals force. The interaction potential in this region, $V_{3}(x)=-C_{3} / x^{3}$, was found by Lennard-Jones 2]. Casimir and Polder included retardation effects which result in the interaction potential $V_{4}(x)=-C_{4} / x^{4}$ at separations of about $1 \mu \mathrm{m}$. In the intermediate region the quantitative description of the van der Waals-Casimir-Polder force invites for the inclusion of material properties. Thermal effects come into play at separations larger than about $2 \mu \mathrm{m}$. Both material properties and thermal effects were included in the Lifshitz theory of the van der Waals forces between dielectrics [3]. This theory describes the case of two parallel plates (separated by a vacuum gap of width $d$ ) and that of an atom at a separation $d$ from a wall.

For a long time the study of atom-wall interaction was considered as merely an academic exercise because the interaction is relatively small. The situation changed radically during the last decade when the atom-wall interaction in different physical, chemical and biological processes received increasing attention [4]. As examples, this interaction plays major role in experimental studies of quantum reflection, Bose-Einstein condensation and diffraction of atoms on different surfaces $[5,6,6,[8,[9,10,11]$. It attracts additional interest in nanotechnological applications, such as carbon nanotubes [12, 13], and mesoscopic-scale atomic devices. All these applications require accurate characterization of atomwall interaction including the dependence of the force on atomic and material properties and on the temperature (see [14, 15] for the most precise computations on the basis of the Lifshitz theory and for a history of the prob-

\footnotetext{
*on leave from North-West Technical University, St.Petersburg, Russia

$\dagger$ on leave from Noncommercial Partnership "Scientific Instru-
} ments", Moscow, Russia lem). In parallel with the Casimir-Polder interaction, the Casimir force between two macrobodies [16] was investigated both experimentally and theoretically (see reviews [17, 18] and recent experiments [19, 20]). Similar to the Casimir-Polder force, the Casimir force finds multidisciplinary applications ranging from constraints on hypothetical interactions predicted by multi-dimensional physics [19] to nanotechnological applications 21. Comparison of the Casimir force measurements with theory also needs to be done with inclusion of real material properties and nonzero temperature [18]. The temperature dependence of the Casimir force between two metal plates is the subject of discussion (see, e.g., [22, 23] ). Some of the measurements of the Casimir force between metallic macrobodies performed up to date [19] were precise enough to exclude theoretical models which take into account the relaxation processes in the real current of conduction electrons. Until now, however, experiments were not of sufficient precision to measure the thermal effect in situ.

The first measurement of the thermal Casimir-Polder force was performed in the excellent experiment 24] both in thermal equilibrium and in the nonequilibrium case. In that experiment the dipole oscillations with the frequency $\omega_{0}$ were excited in a ${ }^{87} \mathrm{Rb}$ Bose-Einstein condensate separated by a distance of a few micrometers from a fused-silica substrate (wall). The CasimirPolder force $F_{C P}$ between a rubidium atom and a substrate changes the magnitude of the oscillation frequency making it equal to some $\omega_{x}$. The fractional frequency difference $\gamma_{x}=\left|\omega_{0}-\omega_{x}\right| / \omega_{0}$ was measured and compared with theory 25, 26] at an environment temperature $T_{E}=310 \mathrm{~K}$ and at different substrate temperatures $T_{S}=310 \mathrm{~K}$ (thermal equilibrium) and $479 \mathrm{~K}, 605 \mathrm{~K}$ (out of thermal equilibrium). In all cases excellent agreement between data and theory was demonstrated.

In this paper, we obtain important new information from the measurement data of experiment 24]. Using theory developed in Refs. [25, 26], we recalculate the fractional frequency difference $\gamma_{x}$ by taking into account a nonzero static conductivity of fused silica at experimental temperatures. We emphasize that introduction of such a small but real conductivity will lead to a dras- 
tically different result in the framework of the Lifshitz theory. The obtained theoretical results both in thermal equilibrium and out of thermal equilibrium are in disagreement with the measurement data. At the same time, when we neglect the static conductivity of fused silica in our calculations, we return back to the computational results of Ref. 24] which are in excellent agreement with the data. We have checked that the differences between the theoretical results computed at different $T_{S}$ with the static conductivity of fused silica included and neglected are completely determined by the equilibrium contribution $F_{C P}$ to the total atom-wall force $F\left(x, T_{S}, T_{E}\right)$ [see Eq. (3) below]. The additional contributions $F_{n}\left(x, T_{S}\right)$ and $F_{n}\left(x, T_{E}\right)$ arising in the atom-wall force out of thermal equilibrium are the same, regardless of whether the static conductivity of the substrate material is included. The obtained experimental evidence that the static conductivity of a wall should be neglected in theoretical calculations using the Lifshitz theory is of much interest for the numerous applications of the Casimir-Polder and Casimir forces. It amplifies the measurement results of Ref. [20], demonstrating that to achieve an agreement between experiment and theory in the Casimir interaction of a gold sphere and a semiconductor plate with doping concentartion below critical, the static conductivity of semiconductor should be neglected. Another type of experiments [19, 27, 28, 29] deals with the Casimir interaction between two gold macrobodies. These experiments also demonstrate that theory taking into account the relaxation processes in real current of conduction electrons is inconsistent with measurement data. To achieve an agreement between data and theory, one should neglect the real current of conduction electrons.

As opposed to [19, 20, 27, 28, 29], the above evidence is based on the measurement data related to a dielectric sample. It is the first one for any kind of material which follows from the data at separation distances of a truly thermal regime above $6 \mu \mathrm{m}$ (experiments in Refs. [19, 20, 27, 28, 29] lead to conclusive results at separations of $1 \mu \mathrm{m}$ and less).

In the experiment 24] the unperturbed trap frequency was measured to be $\omega_{0}=2 \pi \times 229 \mathrm{~Hz}$. The separation distance between the trap center of mass and the surface of the substrate $d$ varies from 6.5 to $11 \mu \mathrm{m}$. Now we briefly present the calculation procedure of the perturbed oscillation frequency $\omega_{x}$, as developed in Refs. [25, 26] (see also [30, 31] for some details).

In accordance with the Lifshits theory, the CasimirPolder force between an atom at a separation $x$ above a substrate at a temperature $T$ in thermal equilibrium is given by

$$
\begin{aligned}
& F_{C P}(x, T)=-2 k_{B} T\left[\alpha_{0} r_{0} \int_{0}^{\infty} k^{3} d k \mathrm{e}^{-2 k x}\right. \\
& \left.\quad+\sum_{l=1}^{\infty} \alpha_{l} \int_{0}^{\infty} k d k \mathrm{e}^{-2 q_{l} x} h\left(\xi_{l}, k\right)\right] \\
& h\left(\xi_{l}, k\right)=\left(2 q_{l}^{2}-\frac{\xi_{l}^{2}}{c^{2}}\right) r_{\mathrm{TM}}\left(\mathrm{i} \xi_{l}, k\right)-\frac{\xi_{l}^{2}}{c^{2}} r_{\mathrm{TE}}\left(\mathrm{i} \xi_{l}, k\right) .
\end{aligned}
$$

Here, the dielectric permittivity of the substrate $\varepsilon_{l}=$ $\varepsilon\left(\mathrm{i} \xi_{l}\right)$ and the atomic dynamic polarizability $\alpha_{l}=\alpha\left(\mathrm{i} \xi_{l}\right)$ are calculated at the imaginary Matsubara frequencies, $\xi_{l}=2 \pi k_{B} T l / \hbar$, and the following notations are introduced

$$
\begin{aligned}
& r_{\mathrm{TM}}=\frac{\varepsilon_{l} q_{l}-k_{l}}{\varepsilon_{l} q_{l}+k_{l}}, \quad r_{\mathrm{TE}}=\frac{q_{l}-k_{l}}{q_{l}+k_{l}}, \quad r_{0}=r_{\mathrm{TM}}(0, k), \\
& q_{l}=\sqrt{k^{2}+\frac{\xi_{l}^{2}}{c^{2}}}, \quad k_{l}=\sqrt{k^{2}+\varepsilon_{l} \frac{\xi_{l}^{2}}{c^{2}}} .
\end{aligned}
$$

When the temperature of the substrate $\left(T_{S}\right)$ and of the surrounding environment $\left(T_{E}\right)$ are different, the force acting between an atom and a substrate was obtained in Ref. [26]

$$
F\left(x, T_{S}, T_{E}\right)=F_{C P}\left(x, T_{E}\right)+F_{n}\left(x, T_{S}\right)-F_{n}\left(x, T_{E}\right),
$$

where the nonequilibrium contribution is defined as

$$
\begin{aligned}
& F_{n}(x, T)=-K \int_{0}^{\infty} d \omega \int_{0}^{\infty} d t f(\omega, t) \mathrm{e}^{-\frac{2 \omega t x}{c}}, \\
& f(\omega, t)=\frac{\omega^{4} t^{2}}{\mathrm{e}^{\frac{\hbar \omega}{k^{T}}}-1}\left[|p(\omega, t)|+\operatorname{Re} \varepsilon(\omega)-1-t^{2}\right]^{1 / 2} \\
& \quad \times\left[\frac{1}{|\sqrt{p(\omega, t)}+\mathrm{i} t|^{2}}+\frac{\left(2 t^{2}+1\right)\left(t^{2}+1+|p(\omega, t)|\right.}{|\sqrt{p(\omega, t)}+\mathrm{i} \varepsilon(\omega) t|^{2}}\right], \\
& K=\frac{2 \sqrt{2} \hbar \alpha_{0}}{\pi c^{4}}, \quad p(\omega, t) \equiv \varepsilon(\omega)-1-t^{2} .
\end{aligned}
$$

The frequency shift of the condensate oscillations under the influence of the force $F$ is obtained as [26]

$$
\begin{aligned}
\omega_{0}^{2}-\omega_{x}^{2}= & -\frac{\omega_{0}}{\pi a m} \int_{0}^{2 \pi / \omega_{0}} d \tau \cos \left(\omega_{0} \tau\right) \\
& \times \int_{-R_{x}}^{R_{x}} d \tilde{x} n_{0}^{x}(\tilde{x}) F\left[d+\tilde{x}+a \cos \left(\omega_{0} \tau\right), T_{S}, T_{E}\right]
\end{aligned}
$$

In the experiment 24] $a=2.50 \mu \mathrm{m}$ is the amplitude of the oscillations, $R_{x}=2.69 \mu \mathrm{m}$ is the Thomas-Fermi radius in the $x$-direction, $m=1.443 \times 10^{-25} \mathrm{~kg}$ is the mass or rubidium atom and

$$
n_{0}^{x}(\tilde{x})=\frac{15}{16 R_{x}}\left(1-\frac{\tilde{x}^{2}}{R_{x}^{2}}\right)^{2} .
$$

As a supplementary element to Refs. [25, 26, 30, 31], we perform analytically the averaging procedures. Substituting Eqs. (3) and (6) into Eq. (5) and integrating 
with respect to $\tilde{x}$ and $\tau$, one arrives at

$$
\gamma_{x}=\frac{1}{m a \omega_{0}^{2}}\left|\Phi_{e}\left(d, T_{E}\right)+\Phi_{n}\left(d, T_{S}\right)-\Phi_{n}\left(d, T_{E}\right)\right|,
$$

where

$$
\begin{aligned}
& \Phi_{e}(d, T)=-2 k_{B} T\left[\alpha_{0} r_{0} \int_{0}^{\infty} k^{3} d k \mathrm{e}^{-2 k d} I_{1}(2 k a) g\left(2 k R_{x}\right)\right. \\
& \left.+\sum_{l=1}^{\infty} \alpha_{l} \int_{0}^{\infty} k d k h\left(\xi_{l}, k\right) \mathrm{e}^{-2 q_{l} d} I_{1}\left(2 q_{l} a\right) g\left(2 q_{l} R_{x}\right)\right], \\
& g(z) \equiv \frac{15}{z^{5}}\left[\left(3+z^{2}\right) \sinh z-3 z \cosh z\right],
\end{aligned}
$$

and $I_{1}(z)$ is the Bessel function. The function $\Phi_{n}(d, T)$ is given by Eq. (4) for $F_{n}(x, T)$ where $x$ should be replaced with $d$ and the function $f(\omega, t)$ with

$$
\tilde{f}(\omega, t)=f(\omega, t) I_{1}\left(\frac{2 a \omega t}{c}\right) g\left(\frac{2 R_{x} \omega t}{c}\right) .
$$

Now we are in a position to compute $\gamma_{x}$ under different assumptions of the conductivity of fused silica. Following Ref. [26], the static dynamic polarizability of Rb atoms $\alpha_{l} \approx \alpha_{0}=4.73 \times 10^{-23} \mathrm{~cm}^{-3}$ is used in computations. This allows one to obtain highly accurate results at separations under consideration [14]. For fused silica $\varepsilon\left(\mathrm{i} \xi_{l}\right)$ as a function of $\xi_{l}$ is taken from [15].

The computational results for $\gamma_{x}$ in thermal equilibrium are shown in Fig. 1 by the solid line. In the same figure, the experimental data obtained in Ref. [24] at separations below $10 \mu \mathrm{m}$ are shown as crosses. The absolute errors in the measurement of separations and $\gamma_{x}$ are presented in true scales at each individual data point. The solid line in Fig. 1 practically coincides with the solid line in Fig. 4(a) of Ref. 24] computed using $\varepsilon\left(\mathrm{i} \xi_{l}\right)=\varepsilon_{0}=3.81$ (only minor deviations are observed at $d<8 \mu \mathrm{m}$ ). Note that in the theory of Refs. [25, 26, 30] the frequencydependent $\varepsilon$ of fused silica was used with a finite static value $\varepsilon_{0}$. It was shown that the account of the frequency dependence leads to only a minor effect and only at small distances. We confirm this conclusion. The theoretical computations are in excellent agreement with the data, as was stated in Ref. 24]. Our computation results for $\gamma_{x}$ in nonequilibrium situations are presented by the solid lines in Fig. 2(a) for $T_{S}=479 \mathrm{~K}$ and in Fig. 2(b) for $T_{S}=605 \mathrm{~K}$. These lines also practically coincide with respective lines in Fig. 4(a) of Ref. [24]. According to [26, 30] the frequency dependence of $\varepsilon(\omega)$ does not affect the nonequilibrium contributions $\Phi_{n}$ in the studied ranges of $d$ and $T$. It is seen that at nonequilibrium the data are also in a very good agreement with theoretical computations.

At nonzero temperature any dielectric possesses nonzero static conductivity. It can be taken into account by replacing the dielectric permittivity $\varepsilon(\omega)$ with

$$
\tilde{\varepsilon}(\omega)=\varepsilon(\omega)+\mathrm{i} \frac{4 \pi \sigma_{0}(T)}{\omega} .
$$

The inclusion of conductivity dramatically affects the calculation results using Eqs. (77), (8). In the above computations $r_{0}=\left(\varepsilon_{0}-1\right) /\left(\varepsilon_{0}+1\right)$ was used following from Eq. (2) for $\varepsilon(0)=\varepsilon_{0}$. The same Eq. (2) with the use of dielectric permittivity (10) results in $r_{0}=1$. This alone changes the contribution from $\Phi_{e}(d, T)$ defined in Eq. (8) and leads to the corresponding change in the magnitudes of $\gamma_{x}$ computed using Eq. (77). We emphasize that this change does not depend on the value of $\sigma_{0}$, but only on the fact that it is nonzero. At $T_{S}=T_{E}=310 \mathrm{~K}$ the conductivity of fused silica sample varies within a wide region from $10^{-9} \mathrm{~s}^{-1}$ to $10^{2} \mathrm{~s}^{-1}$ depending on the concentration of impurities [32, 33]. This results in negligibly small additions to $\varepsilon_{l}=\varepsilon\left(\mathrm{i} \xi_{l}\right)$ at all $\xi_{l} \neq 0$.

The computational results for $\gamma_{x}$ using Eqs. (7), (8) and (10) in thermal equilibrium are shown in Fig. 1 as the dashed line. As is seen in the figure, the first two experimental points are in clear disagreement with theory taking into account the conductivity of fused silica.

In the nonequilibrium situation the disagreement between the experimental data and theory taking the static conductivity of silica into account widens. Direct computations show that the addition of conductivity does not influence the nonequilibrium contributions into $\gamma_{x}$. The magnitudes of $\Phi_{n}(d, T)$ computed with different values of $\sigma_{0}$ from 0 to $10^{3} \mathrm{~s}^{-1}$ coincide up to 6 significant figures. Thus, the conductivity influences only through the equilibrium term $\Phi_{e}(d, T)$. The respective results for $\gamma_{x}$ are presented in Fig. 2 by the dashed lines. As is seen in Fig. $2(\mathrm{a})\left(T_{S}=479 \mathrm{~K}\right)$, the three experimental points exclude the dashed line and the other two only touch it. The dashed line in Fig. 2(b) $\left(T_{S}=605 \mathrm{~K}\right)$ demonstrates that all data exclude the theoretical prediction incorporating the static conductivity of fused silica. Thus, the confidence at which the theoretical approach based on Eq. (10) is excluded by data increases with the increase of substrate temperature $T_{S}$. This is in accordance with the conclusion of Ref. [24] that the Casimir-Polder force for a $605 \mathrm{~K}$ substrate is nearly 3 times larger than for a $310 \mathrm{~K}$ substrate. The comparison of the complete set of data, as given by crosses in Figs. 1 and 2(a,b), with the dashed lines shows that the inclusion of the static conductivity of fused silica in computations of the CasimirPolder force is inconsistent with the experimental data of Ref. [24].

To conclude, we have shown that the inclusion of a small, but physically real, static conductivity for the dielectric substrate in the Lifshitz theory leads to a large increase in the magnitude of the Casimir-Polder force. However, as is shown above, the theoretical predictions including the static conductivity of the dielectric are in a deep disagreement with the experimental data of Ref. 24] for the thermal Casimir-Polder force between an atom and a silica surface. Neglecting the static conductivity of silica in the Lifshitz theory leads to an excellent agreement with the data. Thus, the static conductivity of dielectrics should not be included in the model of the dielectric response. This conclusion is consistent with 
a related but different experiment of the Casimir force measurement between a semiconductor plate and a gold sphere [20], where it was found that the static conductivity of the semiconductor plate with doping concentration below the critical should be neglected. The obtained results clarify the use of the Lifshitz theory and are of wide applicability in all multidisciplinary applications of dis- persion forces.

The authors are greatly indebted to M. Antezza and R. J. Wild for helpful discussions and providing details of calculation procedure used in Ref. [24]. The discussions with G. Bimonte, C. Henkel and U. Mohideen are also acknowledged. This work was supported by the DFG Grant No. 436 RUS 113/789/0-3.
[1] H. B. G. Casimir and D. Polder, Phys. Rev. 73, 360 (1948).

[2] J. E. Lennard-Jones, Trans. Faraday Soc. 28, 333 (1932).

[3] E. M. Lifshitz, Zh. Eksp. Teor. Fiz. 29, 94 (1956) [Sov. Phys. JETP 2, 73 (1956)].

[4] V. A. Parsegian, Van der Waals Forces (Cambridge University Press, Cambridge, 2005).

[5] R. E. Grisenti, W. Schollkopf, J. P. Toennies, G. C. Hegerfeldt, and T. Kohler, Phys. Rev. Lett. 83, 1755 (1999).

[6] F. Shimizu, Phys. Rev. Lett. 86, 987 (2001).

[7] V. Druzhinina and M. DeKieviet, Phys. Rev. Lett. 91, 193202 (2003).

[8] Y. Lin, I. Teper, C. Chin, and V. Vutelić, Phys. Rev. Lett. 92, 050404 (2004).

[9] T. A. Pasquini, Y. Shin, C. Sanner, M. Saba, A. Schirotzek, D. E. Pritchard, and W. Ketterle, Phys. Rev. Lett. 93, 223201 (2004).

[10] H. Oberst, Y. Tashiro, K. Shimizu, and F. Shimizu, Phys. Rev. A 71, 052901 (2005).

[11] D. M. Harber, J. M. Obrecht, J. M. McGuirk, and E. A. Cornell, Phys. Rev. A 72, 033610 (2005).

[12] E. V. Blagov, G. L. Klimchitskaya, and V. M. Mostepanenko, Phys. Rev. B 71, 235401 (2005); ibid. 75, 235413 (2007).

[13] M. Bordag, B. Geyer, G. L. Klimchitskaya, and V. M. Mostepanenko, Phys. Rev. B 74, 205431 (2006).

[14] J. F. Babb, G. L. Klimchitskaya, and V. M. Mostepanenko, Phys. Rev. A 70, 042901 (2004).

[15] A. O. Caride, G. L. Klimchitskaya, V. M. Mostepanenko, and S. I. Zanette, Phys. Rev. A 71042901 (2005).

[16] H. B. G. Casimir, Proc. K. Ned. Akad. Wet. 51, 793 (1948).

[17] M. Kardar and R. Golestanian, Rev. Mod. Phys. 71, 1233 (1999).

[18] M. Bordag, U. Mohideen, and V. M. Mostepanenko,
Phys. Rep. 353, 1 (2001).

[19] R. S. Decca, D. López, E. Fischbach, G. L. Klimchitskaya, D. E. Krause, and V. M. Mostepanenko, Phys. Rev. D 75, 077101 (2007).

[20] F. Chen, G. L. Klimchitskaya, V. M. Mostepanenko, and U. Mohideen, Phys. Rev. B 76, 035338 (2007).

[21] E. Buks and M. L. Roukes, Phys. Rev. B 63, 033402 (2001).

[22] V. B. Bezerra, R. S. Decca, E. Fischbach, B. Geyer, G. L. Klimchitskaya, D. E. Krause, D. López, V. M. Mostepanenko, and C. Romero, Phys. Rev. E 73, 028101 (2006).

[23] J. S. Høye, I. Brevik, J. B. Aarseth, and K. A. Milton, J. Phys. A: Math. Gen. 39, 6031 (2006).

[24] J. M. Obrecht, R. J. Wild, M. Antezza, L. P. Pitaevskii, S. Stringari, and E. A. Cornell, Phys. Rev. Lett. 98, 063201 (2007).

[25] M. Antezza, L. P. Pitaevskii, and S. Stringari, Phys. Rev. A 70, 053619 (2004).

[26] M. Antezza, L. P. Pitaevskii, and S. Stringari, Phys. Rev. Lett. 95, 113202 (2005).

[27] S. K. Lamoreaux, Phys. Rev. Lett. 78, 5 (1997).

[28] R. S. Decca, E. Fischbach, G. L. Klimchitskaya, D. E. Krause, D. López, and V. M. Mostepanenko, Phys. Rev. D 68, 116003 (2003).

[29] R. S. Decca, D. López, E. Fischbach, G. L. Klimchitskaya, D. E. Krause, and V. M. Mostepanenko, Ann. Phys. (N.Y.) 318, 37 (2005).

[30] M. Antezza, J. Phys. A: Math. Gen. 39, 6117 (2006).

[31] L. P. Pitaevskii, J. Phys. A: Math. Gen. 39, 6665 (2006).

[32] Material Science and Engineering Handbook, 3rd ed., edited by J. F. Shackelford and W. Alexander (CRC Press, Boca Raton, 2001).

[33] N. P. Bansal and R. H. Doremus, Handbook of Glass Properties (Academic Press, New York, 1986). 
Figures 


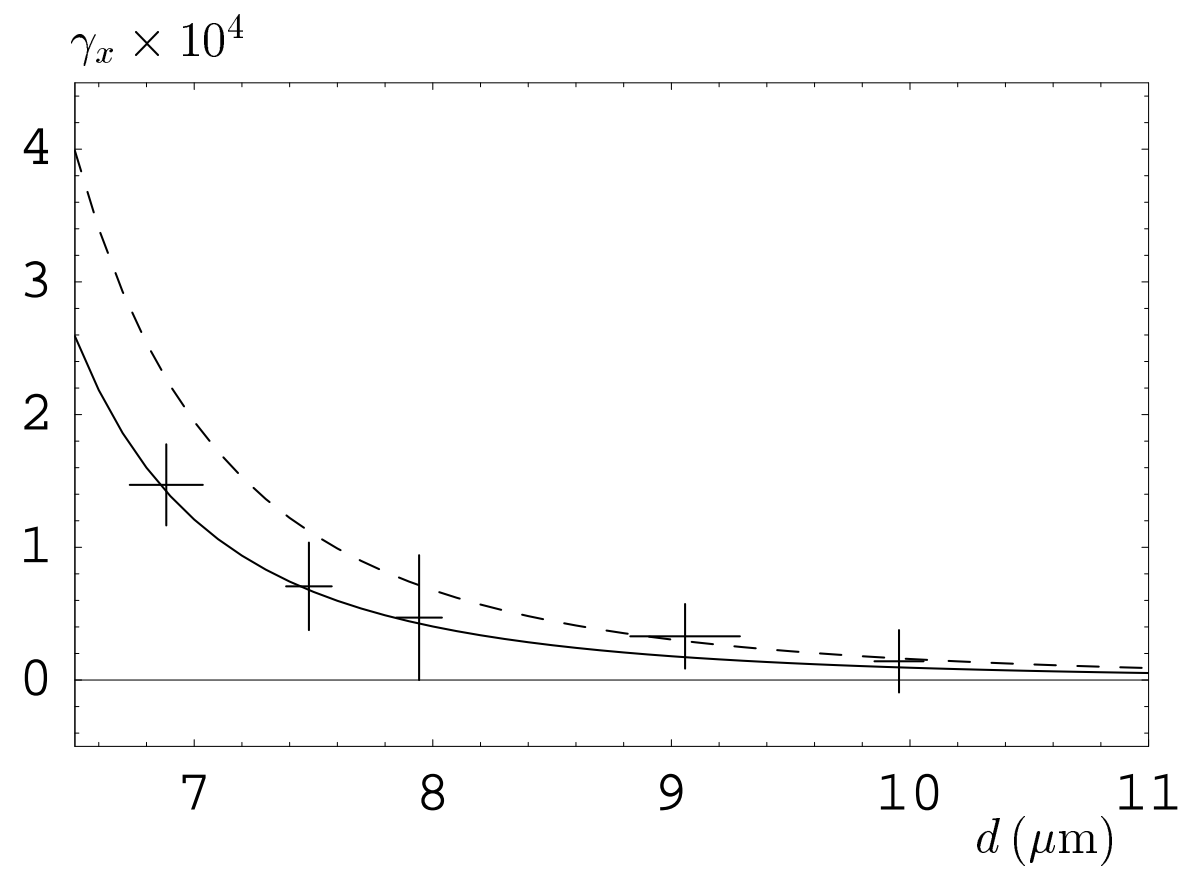

FIG. 1: Fractional change in the trap frequency versus separation in thermal equilibrium with $T_{S}=T_{E}=310 \mathrm{~K}$ computed by neglecting (solid line) and including (dashed line) the conductivity of the dielectric substrate. The experimental data are shown as crosses. 

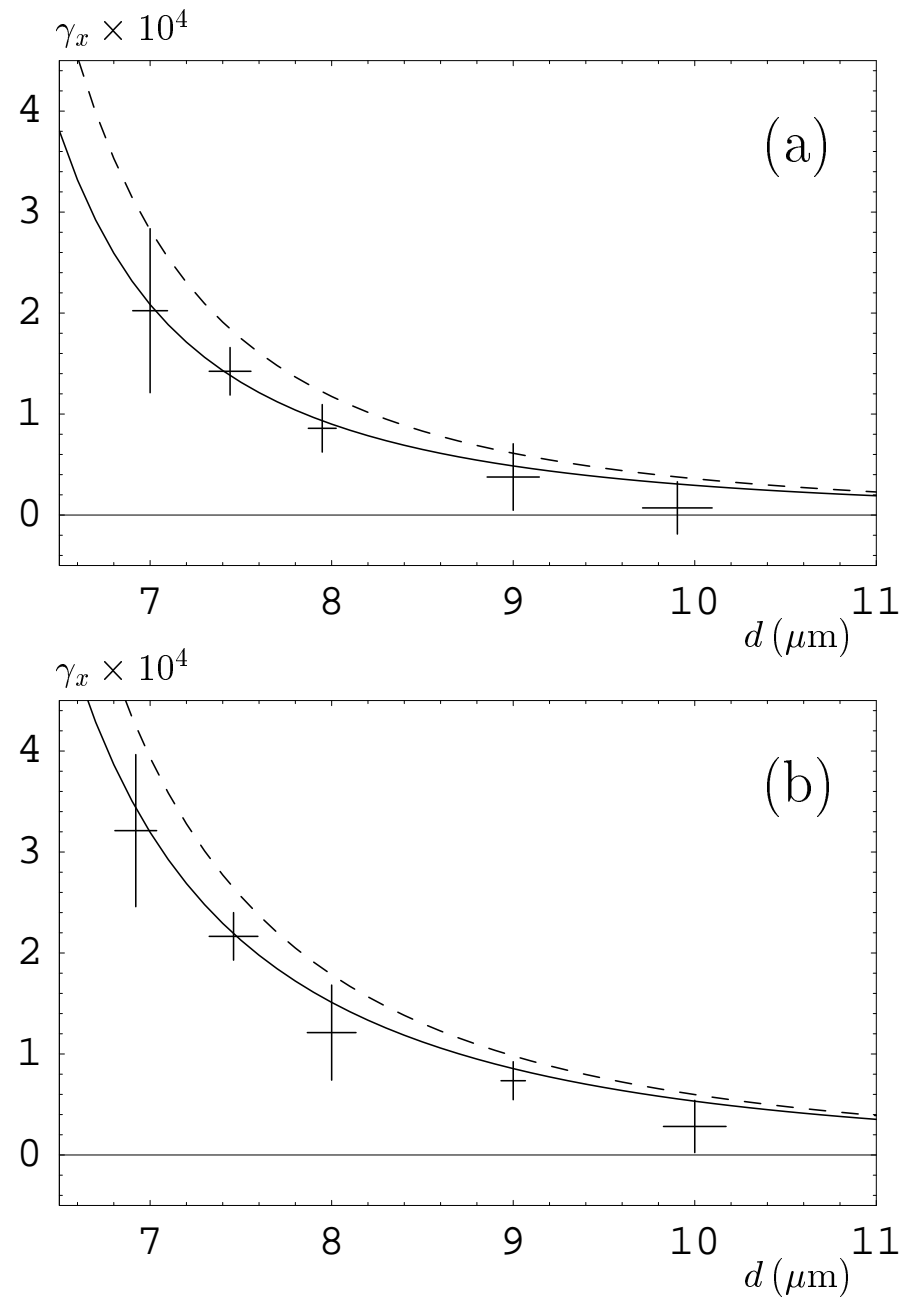

FIG. 2: Fractional change in the trap frequency versus separation out of thermal equilibrium (a) with $T_{S}=479 \mathrm{~K}$ and $T_{E}=310 \mathrm{~K}$ and (b) $T_{S}=605 \mathrm{~K}, T_{E}=310 \mathrm{~K}$. Computations are done by neglecting (solid line) and including (dashed line) the conductivity of the dielectric substrate. The experimental data are shown as crosses. 